\title{
Compilation of Function Representations for Secure Computing Paradigms
}

\author{
Karim Baghery $^{1}{ }^{(0}$, Cyprien Delpech de Saint Guilhem ${ }^{1}$, Emmanuela \\ Orsini $^{1}$ (D) , Nigel P. Smart ${ }^{1,2}$ (D) , and Titouan Tanguy ${ }^{1}$ \\ 1 imec-COSIC, KU Leuven, Leuven, Belgium. \\ ${ }^{2}$ University of Bristol, Bristol, UK. \\ karim.baghery@kuleuven.be, cyprien.delpechdesaintguilhem@kuleuven.be, \\ emmanuela.orsini@kuleuven.be, nigel.smart@kuleuven.be, \\ titouan.tanguy@kuleuven. be
}

\begin{abstract}
This paper introduces M-Circuits, a program representation which generalizes arithmetic and binary circuits. This new representation is motivated by the way modern multi-party computation (MPC) systems based on linear secret sharing schemes actually operate. We then show how this representation also allows one to construct zero knowledge proof (ZKP) systems based on the MPC-in-the-head paradigm. The use of the M-Circuit program abstraction then allows for a number of program-specific optimizations to be applied generically. It also allows to separate complexity and security optimizations for program compilation from those for application protocols (MPC or ZKP).
\end{abstract}

\section{Introduction}

Secure computation methodologies are becoming more mainstream with multiparty computation (MPC), fully homomorphic encryption (FHE) and zero-knowledge proofs of knowledge (ZKPoKs) all finding applications at an increasing rate. At their heart all three technologies work with a public function; either to be compute it securely (in the case of MPC or FHE), or to prove the correctness of public outputs under secret inputs (in the case of ZKPoKs). The representation of this function is key to many of the practical realizations. For example: in theoretical MPC papers functions are often represented by arithmetic circuits, in FHE they are often binary circuits, and in ZKPoK papers R1CS representations are often used.

In this work we concentrate on two secure computation technologies: MPC protocols based on linear secret sharing schemes (LSSS) and ZKPoKs based on MPC-in-the-Head (MPCitH). It is common in theoretical treatments of these protocols to assume the input function representation is given as an arithmetic or binary circuit. However, in practice, this is not how functions are represented as input to such protocols. It has been known since Beaver's work [6] that sometimes a more interesting representation is as a set of linear operations, combined with a correlated randomness source. Previous work showed that more 
efficient representations for LSSS-based MPC can be obtained using different sources of correlated randomness, as well as a combination of different finite fields $[10,11,16]$. This latter idea has been expanded in recent years with the advent of so-called daBit-based protocols for switching between LSSS-based MPC and garbled circuit-based MPC [27]. Thus the standard theoretical assumption of representing the function as a simple arithmetic circuit is at least ten years out of date given the state-of-the-art of LSSS-based MPC protocols.

A similar situation holds for MPCitH protocols. These are a class of zeroknowledge protocols introduced by Ishai et al in [22], and recently extended in a number of works eg. $[3,5,9,12,21,23]$ In such protocols, a key aspect is to represent the function as a sequence of linear operations, combined with access to sources of correlated randomness, as in [5,23]. In MPC protocols, the creation of the correlated randomness sources often involves expensive pre-processing, but for MPCitH this can be done essentially for free. Therefore, larger performance improvements for MPCitH could result from expanding the use of correlated randomness sources.

Indeed in both LSSS-based MPC and MPCitH there is no single 'correct' representation of a function, with different representations presenting different performance trade-offs in the final protocol. But it is also case that different representations can also present different security trade-offs as well. Indeed the compilation of the abstract function into a concrete representation can introduce security issues.

Our Contributions and Paper Overview. The first contribution of this paper (in Section 2) is a generalized definition of the program input to such LSSS-based MPC and MPCitH protocols, which we call an M-Circuit. It can be considered as a generalization of arithmetic circuits, but tuned for MPC and MPCitH protocols (hence the name). An M-Circuit can make use of linear operations on sensitive variables, correlated randomness sources, as well as objects we call 'gadgets'. A gadget is a function call from the M-Circuit representation of the program which is not necessarily implemented itself as an M-Circuit. Such gadgets allow for specific functions to be implemented in ways which avoid inefficiencies from implementing them using only an M-Circuit definition. Each M-Circuit belongs to a set of classes of M-Circuits, determined by what correlated randomness sources we allow, what finite fields are utilized, and what magic 'gadgets' are used. We can then determine which classes of protocols are best suited for different protocols.

We furthermore examine what it means for a compilation of a function into an M-Circuit to be 'secure'. And we relate this security definition to the security of the resulting MPC/MPCitH protocol when the given M-Circuit representation is used. Some compilation strategies are clearly insecure, some give perfect security and some give statistical security; leading to the same corresponding security of the final protocol in which they are used. Whilst well understood in the practical community, we can find no treatment of the security aspect of program compilation being discussed in the MPC literature before. 
After presenting our representation we show how this maps, in Section 3, onto common MPC frameworks (such as MP-SPDZ [24] and SCALE-MAMBA [1]), and how the M-Circuit representation is already the underlying one used in practice. This application is now standard and we only sketch it in this work.

In our second contribution in Section 4, we show how M-Circuits can be used in MPCitH protocols. We recast the protocols of $[5,23]$ to use our general M-Circuit definition (initially excluding the use of the gadgets). We define the components needed to allow M-Circuits to be used in general MPCitH protocols. We then go on to present a number of optimization strategies which our M-Circuit representation allows one to express easily for MPCitH protocols. The first, in Section 5, examines how introducing new correlated randomness sources can produce more efficient proofs. We recall that for MPCitH adding new correlated randomness sources comes at little extra cost and thus this is a resource we can utilize to improve efficiency quite aggressively. The second, in Section 6 , shows how, for some randomness sources, one can replace cut-and-choose checking with a form of sacrificing (as used in MPC protocols such as SPDZ). This acts as a warm up for our method which introduces the ability to introduce complex gadgets into our MPCitH protocol, given in Section 7. Details of standard definitions and proofs of our main results are given in the full version.

\section{M-Circuits}

Given a function $F$ there are many ways of representing the function: theoreticians may look at binary or arithmetic circuit representations, programmers may think of C, Java, or Haskell, a processor designer may think of an x86 instruction stream. By the Church-Turing thesis all are essentially equivalent. In this section we formalize a way of representing a function for use in LSSS-based MPC and/or MPCitH systems. A key aspect of our definition is that the process of compiling/programming an abstract mathematical function $F$ as a concrete representation involves some form of security analysis, i.e. it is not only the MPC/MPCitH protocol which impacts security but also the input representation of the function being operated upon.

\subsection{Defining an M-Circuit}

At the heart of our definitions is the idea that a function maps input variables to output variables, but that some of the input variables, and indeed some of the output variables may be sensitive.

Machine State. We start by defining a machine state.

Definition 2.1 (Machine State). A machine state state defined over a set of finite fields $\mathcal{F}=\left\{\mathbb{F}_{q_{1}}, \ldots, \mathbb{F}_{q_{f}}\right\}$ is a collection of variables (or memory addresses). Each variable has a type which is one of the following three forms: 
- $\left(q_{i},-\right)$, which refers to a variable which holds a non-sensitive variable in the finite field $\mathbb{F}_{q_{i}}$;

- $\left(q_{i}, s\right)$, which refers to a variable which holds a sensitive variable in the finite field $\mathbb{F}_{q_{i}}$;

- $(-,-)$ which refers to a signed integer variable (i.e. an element of $\mathbb{Z})$ in some bounded range (for example a 64-bit integer).

The machine state can hold these variables in a number of manners, for example as memory locations indexed by integers via stacks. The usage of the signed integer variables are to allow memory access operations and stack operations within the machine. Note, one could extend the definition to finite rings, and not just finite fields, using techniques such as those from SPDZ2k [15], but for now we keep to the simpler case of finite fields.

To ease notation in what follows we let $\{x\}_{q}$ denote a variable of type $(q,-)$, $\langle x\rangle_{q}$ denote a variable of type $(q, s)$, and $x$ denote a variable of type $(-,-)$. Also, we make no distinction between the name of the variable and the value it contains. If we want to refer to a type, and are not interested in its sensitivity classification, we refer to the type $(q, *)$, and call it the base type of the variable.

We note that variables of the same type can be added, subtracted and multiplied etc. Variables of different types can be combined in the following sense: the operation of a binary arithmetic operator on two variables of type $\left(p_{1}, s_{1}\right)$ and $\left(p_{2}, s_{2}\right)$ can be applied if $\operatorname{gcd}\left(p_{1}, p_{2}\right) \neq 1$, resulting in a type $\left(p_{3}, s_{3}\right)$ where:

- $p_{3}=\operatorname{gcd}\left(p_{1}, p_{2}\right)$,

- $s_{3}=s$ if and only if $s_{1}=s$ or $s_{2}=s$, otherwise $s_{3}=-$. (This means that variables can only become more sensitive, akin to the 'no write down' rule of Bell-LaPadula [7]).

Thus one can form (relatively) arbitrary arithmetic expressions on variables, and one can assign a type to the result of the expression.

Variables of type $(p,-)$, for prime $p$, can be arbitrarily converted into variables of type $(-,-)$ and vice-versa, using the inclusion $\mathbb{F}_{p} \longrightarrow[0, \ldots, p-1) \subset \mathbb{Z}$ and the mapping $\mathbb{Z} \longrightarrow \mathbb{F}_{p}$ given by $x \mapsto x(\bmod p)$.

Correlated Randomness Sources: As well as variables, and the arithmetic expressions we can create from them, there are two additional components for an M-Circuit, namely correlated randomness sources and gadgets, which we describe in the following.

Definition 2.2 (Correlated Randomness Source). A correlated randomness source $\mathbb{S}$ is defined by a set of variables $\left\{v_{1}, \ldots, v_{t}\right\}$ of any (specific) types $\left\{\left(q_{1}, *\right), \ldots,\left(q_{t}, *\right)\right\}$ and a predicate pred on those variables.

A correlated randomness source should be thought of as related to the data which is produced in preprocessing phases of MPC protocols such as SPDZ [20]. Thus typical sources would be: 
- Triple: This has associated to it three variables, $(a, b, c)$ all of type $(p, s)$, for which the predicate is $\operatorname{pred}(a, b, c):=a \cdot b=c$, with $a, b$ being uniformly randomly chosen from $\mathbb{F}_{p}$.

- Square: This has associated to it two variables, $(a, b)$, both of type $(p, s)$ for which the predicate is $\operatorname{pred}(a, b):=a \cdot a=b$, with $a$ being uniformly randomly chosen from $\mathbb{F}_{p}$.

- Bit: This has associated to it a single variable, $a$, of type $(p, s)$ for which the predicate is $\operatorname{pred}(a):=a \in\{0,1\}$, and $a$ is uniformly randomly chosen from $\{0,1\}$.

- daBit: This has associated to it two variables, $a, b$, one of type $(p, s)$ and one of type $(2, s)$, for which the predicate is $\operatorname{pred}(a, b):=(a=b) \wedge(a \in\{0,1\})$, with $a$ uniformly randomly chosen from $\{0,1\}$.

Gadgets: The second component we introduce now is called a 'gadget'. From a high level point of view these can be arbitrary operations. More formally, they are function calls made by the M-Circuit which we do not necessarily implement using an M-Circuit. This means, for example, that their functionality could be provided by some externally defined protocol. In practice, we will use gadgets to perform very specific operations within specific protocols and also to help to define stages of program transformation within a compilation. Thus gadget's correspond to operations which are done using special protocols, with the idea being that if we can show the special protocol for implementing the gadget is secure and correct, then we can use the gadget as an optimization process within our final protocols.

Definition 2.3 (Gadget). A gadget $\mathbb{G}$ is a mathematical function which takes a set of variables and outputs a set of variables $\left(\hat{v}_{1}, \ldots, \hat{v}_{u}\right) \leftarrow \mathbb{G}\left(v_{1}, \ldots, v_{t}\right)$, where no assumption is made about how $\mathbb{G}$ will be implemented. The types of the input and output variables are assumed to be implicitly defined by the gadget itself.

Looking ahead, in the context of MPC using a gadget is like calling a protocol to perform a Garbled Circuit operation on some secret shared data over $\mathbb{F}_{2}$ in a system such as that described by the Zaphod paper [2]. The gadget in this case goes outside the neat confines of LSSS-based MPC, but it is integrated with the LSSS based MPC and is thus able to allow greater functionality at reduced cost. Another example of a gadget could be a multiplication gate, which we do not expand into its Beaver representation if we want to avoid correlated randomness sources.

Instructions and M-Circuits. An M-Circuit is composed of an ordered finite list of instructions as follows.

Definition 2.4 (Instruction). An instruction can be one of the following forms: 
- A pair ( $v$, expr), where $v$ is a variable and expr, is an arithmetic expression as described above. The type of $v$ must correspond to the type of expr. As a shorthand we may write $v \leftarrow$ expr. We restrict the expressions expr to be arbitrary arithmetic expressions, however the total degree of the expression in any sensitive variables must be one. Thus we can only compute linear functions on sensitive variables.

- A tuple $\left(\left\{v_{1}, \ldots, v_{t}\right\}, \mathbb{S}\right)$ where $\mathbb{S}$ is a correlated randomness source, and the variables $\left\{v_{1}, \ldots, v_{t}\right\}$ have the same types as the variables associated to the source. As a shorthand we may write $v_{1}, \ldots, v_{t} \leftarrow \mathbb{S}$.

- A tuple $\left(\left\{\hat{v}_{1}, \ldots, \hat{v}_{u}\right\},\left\{v_{1}, \ldots, v_{t}\right\}, \mathbb{G}\right)$ where $\hat{v}_{i}$ and $v_{i}$ are variables and $\mathbb{G}$ is a gadget as described above. The types of $v_{j}$ and $\hat{v}_{i}$ must correspond to the input and output types of the Gadget. As a shorthand we may write $\hat{v}_{1}, \ldots, \hat{v}_{u} \leftarrow \mathbb{G}\left(v_{1}, \ldots, v_{t}\right)$.

- A 'declassification' instruction which we write as $x \leftarrow y$.reveal(). This takes a variable $y$ of type $(p, s)$ and creates a variable $x$ of type $(p,-)$ which has the same value as $y$.

- A special instruction called terminate.

Examples, of the first three types of instruction, could include:

$$
\begin{aligned}
\langle z\rangle & \leftarrow\langle x\rangle_{p}+\langle y\rangle_{p} \\
\langle x\rangle_{p},\langle y\rangle_{p},\langle z\rangle_{p} & \leftarrow \text { Triple } \\
\left\{\left\langle c_{i}\right\rangle_{2}\right\}_{i=0}^{127} & \leftarrow \operatorname{AES}\left(\left\{\left\langle k_{i}\right\rangle_{2}\right\}_{i=0}^{127},\left\{\left\langle m_{i}\right\rangle_{2}\right\}_{i=0}^{127}\right) .
\end{aligned}
$$

Finally, we can define what we mean by an M-Circuit.

Definition 2.5. An M-Circuit is a tuple consisting of an ordered list of instructions $\mathcal{I}$ and two sets of variables $\mathcal{V}_{I}$ and $\mathcal{V}_{O}$ (called the input and the output variables).

A class of M-Circuits $\mathcal{C}_{\left\{\mathbb{F}_{q_{1}}, \ldots, \mathbb{F}_{q_{f}}\right\}}^{\left.\left(\left\{\mathbb{S}_{1}, \mathbb{S}_{1}\right\}, \ldots, \mathbb{G}_{g}\right\}\right)}$ is the set of all M-Circuits over the finite fields $\left\{\mathbb{F}_{q_{1}}, \ldots, \mathbb{F}_{q_{f}}\right\}$, which utilize correlated randomness sources $\left\{\mathbb{S}_{1}, \ldots, \mathbb{S}_{s}\right\}$ and gadgets $\left\{\mathbb{G}_{1}, \ldots, \mathbb{G}_{g}\right\}$. If $\mathcal{F}$ and $\mathcal{F}^{\prime}$ are sets of finite fields, and $\mathcal{S}$ and $\mathcal{S}^{\prime}$ are sets of correlated randomness sources, and $\mathcal{G}$ and $\mathcal{G}^{\prime}$ are sets of gadgets then we have $\mathcal{C}_{\mathcal{F}}^{(\mathcal{S}, \mathcal{G})} \subseteq \mathcal{C}_{\mathcal{F}^{\prime}}^{\left(\mathcal{S}^{\prime}, \mathcal{G}^{\prime}\right)}$ if $\mathcal{F} \subseteq \mathcal{F}^{\prime}, \mathcal{S} \subseteq \mathcal{S}^{\prime}$ and $\mathcal{G} \subseteq \mathcal{G}^{\prime}$.

\subsection{Executing an M-Circuit}

An M-Circuit $\left(\mathcal{I}, \mathcal{V}_{I}, \mathcal{V}_{O}\right)$ operates on a machine state as follows. The machine state state has an initial state consisting of the set of registers $\mathcal{V}_{I}$ with preassigned values given to them (i.e. the inputs to the function). In addition there is a special register of type $(-,-)$, called $\mathrm{pc}$, which is initial set to zero. Then the following operations are repeated until a terminate instruction is met.

- Instruction numbered $\mathrm{pc}$ is fetched from the list of instructions $\mathcal{I}$.

- The value of $\mathrm{pc}$ is incremented by one. 
- The instruction is executed as follows depending on its type

- $(v$, expr $)$ is evaluated if all the variables in expr are currently defined, and the result is assigned to variable $v$. If not all variables are defined then the system aborts.

- $\left(\left\{v_{1}, \ldots, v_{t}\right\}, \mathbb{S}\right)$ is evaluated by sampling the variables $\left\{v_{1}, \ldots, v_{t}\right\}$ according to the source definition.

- $\left(\left\{\hat{v}_{1}, \ldots, \hat{v}_{u}\right\},\left\{v_{1}, \ldots, v_{t}\right\}, \mathbb{G}\right)$ is evaluated as for $(v$, expr $)$

- Declassification instructions do the obvious declassification operation.

- If the instruction is terminate then the M-Circuit terminates.

On termination the M-Circuit outputs the variables in the set $\mathcal{V}_{O}$, if they are defined. If any are not defined it aborts.

Note, this is a rather general model in a number of senses:

- One can perform a conditional branch on non-sensitive variables by making instructions of the form (pc, expr), e.g. pc $\leftarrow b \cdot 100+(1-b) \cdot 200$ will either result in a jump to instruction 100 or instruction 200 depending on the value of variable $b \in\{0,1\}$ of type $(-,-)$.

- Subroutines calls, and hence recursion, can be performed by using creating a stack of type $(-,-)$ in the machine state, and then using this to push/pop the $\mathrm{pc}$ variable on or off of it.

The main limitation of the model seems to be that instructions of the form $(v$, expr) can only contain linear functions of sensitive variables. This is where our gadgets and randomness sources will come in.

There are four different measures of complexity of an M-Circuit, and we name these so as to link them with their analogues when we use M-Circuits for MPC (where analogues exist), as follows.

- The offline complexity is the number of calls to the source oracles $\left\{\mathbb{S}_{1}, \ldots, \mathbb{S}_{s}\right\}$ made by the M-Circuit on a given input.

- The online communication complexity is the number of calls to the operation reveal() made by the M-Circuit on a given input.

- The online round complexity is the minimum number of parallel calls to the operation reveal() made by the M-Circuit on a given input.

- The gadget-complexity (which has no usual analogue in the MPC domain) is the number of calls to gadgets $\mathbb{G}$ made by the M-Circuit on a given input.

\subsection{Compiling M-Circuits}

An M-Circuit is created by a process called compilation.

Definition 2.6 (Compilation). A compilation step is an algorithm which takes an $M$-Circuit $C$ in a class $\mathcal{C}_{\mathcal{F}}^{(\mathcal{S}, \mathcal{G})}$ and maps it to an $M$-Circuit $C^{\prime}$ in a class $\mathcal{C}_{\mathcal{F}^{\prime}}^{\left(\mathcal{S}^{\prime}, \mathcal{G}^{\prime}\right)}$. The algorithm must ensure that the functional behaviour of $C$ and $C^{\prime}$ are identical, i.e. the input/output behaviour of $C$ and $C^{\prime}$ are the same. 
Note a compilation says nothing about whether $\mathcal{C}_{\mathcal{F}}^{(\mathcal{S}, \mathcal{G})} \subseteq \mathcal{C}_{\mathcal{F}^{\prime}}^{\left(\mathcal{S}^{\prime} \mathcal{G}^{\prime}\right)}$ or vice-versa.

Given an arbitrary polynomial time function $F$, defined over a set of finite fields $\mathcal{F}$ and the integers, there is always an M-Circuit, which we call $C_{F}$, which implements $F$ in the class $\mathcal{C}_{\mathcal{F}}^{(\emptyset,\{F\})}$, namely the M-Circuit which uses the gadget $\mathbb{G}=F$. The goal of compilation is to find representations of $C_{F}$ in simpler classes, in particular a class which can be implemented in either an MPC or MPCitH system. We present three exemplar compilations here to fix ideas:

- Arithmetic Circuit: Consider the gadget $\mathbb{G}_{M}$ for a finite field $\mathbb{F}_{p}$ which multiples two input values, giving the output value of the same type. Then the standard 'arithmetization' of polynomial time functions $F$ compiles the M-Circuit $C_{F}$ to an M-Circuit $C_{F}^{A}$ in the class $\mathcal{C}_{\mathbb{F}_{p}}^{\left(\emptyset,\left\{\mathbb{G}_{M}\right\}\right)}$.

- Beaver Randomized Circuit: We can take the M-Circuit $C_{F}^{A}$ produces in the previous example and compile it to an M-Circuit $C_{F}^{B}$ in the class $\mathcal{C}_{\mathbb{F}_{p}}^{(\text {Triple, }\{\emptyset\})}$ using Beaver's standard circuit randomization trick, [6].

- Insecure Circuit: Here we take $F$, and create the functionally equivalent function $F^{\prime}$ which first de-classifies all the sensitive input variables of $F$. Then it evaluates $F$ on the clear values, using the arithmetic circuit representation of $F$. Finally, it re-classifies any output variables as sensitive which need to be sensitive, by multiplication by $\langle 1\rangle_{p}$. The associated arithmetic circuit $C_{F^{\prime}}^{A}$ (which includes reveal() operations as well as the usual arithmetic operations) is an M-Circuit in the class $\mathcal{C}_{\mathbb{F}_{p}}^{\left(\emptyset,\left\{\mathbb{G}_{M}\right\}\right)}$.

The last example here hints that compilation can create something which is 'insecure'. To quantify this notion we need to define what we mean by security of an M-Circuit.

\subsection{Security of M-Circuits}

To define security of an M-Circuit we have to examine the reveal() operations in more detail, since these are the operations which potentially de-classify sensitive information. Informally we require that the reveal() operations never reveal more than an negligible amount of sensitive information about any sensitive inputs to the function.

To each reveal operation of the form $a \leftarrow\langle b\rangle_{q}$.reveal() we associate a given distribution $\mathcal{R}_{b}$ on the set $\mathbb{F}_{q}$. The reader can think of $\mathcal{R}_{b}$ on first reading as the uniform distribution (which will be true for circuits compiled using the Beaver compilation above, but it is not true in general). To take into account the type of efficient function representations used in say [11] we need to be a little more nuanced.

The distributions $\mathcal{R}_{b}$ are on the outputs of reveal() are conditioned on the following three things:

- The specific non-sensitive inputs and outputs of the function being evaluated.

- The random execution path taken by the circuit.

- The distributions of the correlated randomness sources. 
However, the distributions are not conditioned on sensitive input and output values to the function. For example consider the code fragments in Figure 1. In fragment (a) the function is $b$, in which case the distribution $\mathcal{R}_{z}$ has probability mass of one at the value $b-1$ and is zero elsewhere, whilst in fragment (b) the distribution $\mathcal{R}_{z}$ is the set of values in the range $[-B, \ldots, B]$ with an associated binomial distribution.

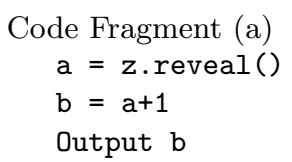

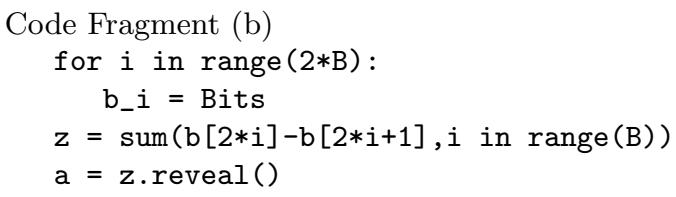

Fig. 1. Example Code Fragments

The trace Trace $_{C}$ of an M-Circuit, on a given input, consists of the set of non-sensitive input variables, the non-sensitive output variables, plus the output of every reveal() operation. A simulated trace $\operatorname{Sim}_{C}$ is the same except that the output of every reveal() operation is replaced by a value chosen via the distributions $\mathcal{R}_{b}$ above.

Definition 2.7 (Perfectly Secure M-Circuit). An M-Circuit $C$ is said to perfectly securely implement a function $F$ if the functional behaviour of the $M$ Circuit $C$ and the $M$-Circuit $C_{F}$ are identical, and the distribution of Trace and $\operatorname{Sim}_{C}$ are identical for all input values.

Definition 2.8 (Statistically Secure M-Circuit). The M-Circuit $C$ is said to securely implement a function $F$ with statistical security sec if the functional behaviour of the $M$-Circuit $C$ and the function $C_{F}$ are identical, and the statistical distance between the distribution of $\operatorname{Trace}_{C}$ and $\mathrm{Sim}_{C}$ is bounded by $2^{-\mathrm{sec}}$ for all input values.

The first question one must ask is if such a definition is vacuous. The celebrated technique of Beaver's Circuit Randomization [6] says no.

Theorem 2.1. Every polynomial time function $F$ can be perfectly securely implemented by the $M$-Circuit $C_{F}^{B}$ with polynomial complexity (in all four metrics).

Proof. Using the compilation process above we can compile the M-Circuit $C_{F}^{B}$. It is well known that the reveal() operations this creates are associated with uniform distributions, and thus the reveals are perfectly hiding. 
Note, this definition is about the representation of the function i.e. the compilation of the M-Circuit from the function definition. It asks whether the compilation process is itself secure; it makes no claim about how the M-Circuit is then used or evaluated within an MPC or MPCitH system.

Not all compilations will result in secure M-Circuits, as our insecure compilation example illustrates. To see why this compilation violates our security definition, consider the specific function $F$ which takes two sensitive values $x$ and $y$, and returns their sum, but as a non-sensitive value. Mathematically one could write $F(\langle x\rangle,\langle y\rangle)=(\langle x\rangle+\langle y\rangle)$.reveal(). A functionally valid M-Circuit for this function is given in code fragment (c) of Figure 1, whilst another functionally valid M-Circuit for the same function is given in code fragment $(\mathrm{d})$.

Code fragment (c) is a perfectly secure M-Circuit, with the distribution $\mathcal{R}_{z}$ being the point distribution will all the probability mass at the point $a$ (where $a$ is the public output of the function). Thus the valid transcript $\operatorname{Trace}_{C}$ and $\operatorname{Sim}_{C}$ are identical and equal to $\operatorname{Trace}_{C}=\operatorname{Sim}_{C}=\{\emptyset,\{a\},\{a\}$.$\} , i.e. there are no$ distributions here at all, Trace ${ }_{C}$ and $\operatorname{Sim}_{C}$ are fixed by the output $a$. Note, the $\emptyset$ corresponds to the set of non-sensitive input variables, the first $\{a\}$ is the set of non-sensitive output variables, and the second $\{a\}$ is the output of every reveal (resp. the simulated reveals) in the case of the actual trace (resp. the simulated trace).

Code fragment (d) has $\mathcal{R}_{x}$ being the point distribution on $x$, with $y$ being the point distribution of $y=a-x$. Thus Trace $_{C}$ of the second M-Circuit is equal to Trace $_{C}=\{\emptyset,\{a\},\{x, a-x\}\}$ which is a fixed value (for each given input), whereas the simulated trace $\mathcal{S}_{C}$ is equal to the value given by $\mathcal{S}_{C}=\{\emptyset,\{a\},\{r, a-r\}\}$ where $r$ uniformly chosen from $\mathbb{F}_{p}$. Thus Trace $C$ and $\mathcal{S}_{C}$ in the second case can never be statistically close. Thus the second compilation to an M-Circuit is insecure, but functionally correct.

\section{M-Circuits for Multi-Party Computation}

It turns out that our M-Circuit notion lies underneath almost all algorithmic level optimizations of LSSS-based MPC over the last decade (by which we mean optimizations related to the program representation and not the MPC protocol itself). The M-Circuit concept allows us to isolate which optimizations can be utilized by which MPC protocols, since not all MPC protocols can implement all M-Circuit classes.

As remarked earlier, arithmetic circuit representation of a functionality over a finite field $\mathbb{F}_{q}$ correspond to M-Circuits in the class $\mathcal{C}_{\mathbb{F}_{q}}^{\left(\emptyset,\left\{\mathbb{G}_{M}\right\}\right)}$. Thus 'traditional' LSSS based MPC protocols such as [8,13], or modern protocols such as [14], which have specific protocols for the multiplication operation can utilize this MCircuit representation. However, the security of these protocols is then proved by showing that the implementation of the specific multiplication gadget leaks no information.

Protocols which expand the multiplication gadget via Beaver's trick [6] utilize circuits from the class $\mathcal{C}_{\mathbb{F}_{p}}^{(\{\text {Triple }, \emptyset)}$. The security of the underlying (passively 
secure) online protocol then follows from the security of the M-Circuit representation; if the M-Circuit is secure then so is the obvious LSSS-based MPC protocol in which one replaces the sensitive variables in the M-Circuit by secret shared values. The problem comes in creating an offline phase to produce the necessary correlated randomness source Triple in a secret shared manner. For honest majority protocols this offline phase is usually performed using hyperinvertible matrices (as in VIFF [17]) or, for dishonest majority protocols using homomorphic encryption (as in SPDZ [20]) or OT (as in MASCOT [26]). In the latter case to prove active security of the underlying MPC protocol one needs to provide a form of authenticated secret sharing, while the privacy of the protocol follows from the security of the M-Circuit representation. For the passive case the security of the online phase we cover in Section 4.1 later.

Papers such as $[10,11,16]$ showed that one can obtain greater efficiency by working with M-Circuits in the class $\mathcal{C}_{\left.\left.\mathbb{F}_{28}, \mathbb{F}_{q}\right\}\right)}^{\left(\emptyset,\left\{\mathbb{F}_{M}\right\}\right.}$, or equivalently $\mathcal{C}_{\mathbb{F}_{28}, \mathbb{F}_{q}}^{\left(\left\{\text {Tripe }_{2}, \text { Triple }_{q}\right\}, \emptyset\right)}$; although of course they did not use this language. In these latter works the authors used multiplication to create shared-random bits, whereas if one assumes these as a random source then the protocols become simpler to describe; thus the same work can be cast as corresponding to M-Circuits in the class $\mathcal{C}_{\mathbb{F}_{q}}^{(\{\text {Triple,Bit }\}, \emptyset)}$. It is this latter representation which is used in modern LSSS-based systems in the pre-processing model; for example the second generation of the SPDZ protocol [18] utilizes function descriptions which are M-Circuits in the class $\mathcal{C}_{\mathbb{F}_{p}}^{(\{\text {Triple,Square,Bit }\}, \emptyset)}$. The papers such as $[10,11]$ also showed one can obtain more efficient representations, in terms of minimizing the various complexity measures we described earlier, by compiling to what we call statistically secure M-Circuits as opposed to perfectly secure M-Circuits.

Systems which make use of daBits [27] to translate between binary and arithmetic fields utilize M-Circuits in the class $\mathcal{C}_{\left\{\mathbb{F}_{p}, \mathbb{F}_{2}\right\}}^{(\{\text {Triple,Square,Bit,daBit }\}, \emptyset)}$. Systems such as Zaphod, [2] extend this idea further by allowing gadgets based on garbled circuits to be evaluated within the MPC-computation. Thus they allow M-Circuits in the class $\mathcal{C}_{\left\{\mathbb{F}_{p}, \mathbb{F}_{2}\right\}}^{\left(\{\text {Triple, Square,Bit,daBit }\},\left\{\mathbb{G}_{1}, \ldots, \mathbb{G}_{g}\right\}\right)}$ for specific garbled circuit based subprocedures $\mathbb{G}_{1}, \ldots, \mathbb{G}_{g}$. As long as the gadget can be securely implemented, then the overall MPC protocol is itself secure.

Obviously compilation methods, and different sources of correlated randomness, will give different M-Circuits with different complexities. This is essentially the engineering challenge of MPC solutions: to pick the compilation strategy and sources of correlated randomness in order to achieve an efficient M-Circuit which can be executed by a given MPC engine.

\section{M-Circuits for MPC-in-the-Head}

In this section, we present an MPCitH-based Honest Verifier Zero-Knowledge (HVZK) argument of knowledge system for satisfiability of a function (computation) that is compiled to an M-Circuit. Initially we consider M-Circuits with no gadgets, namely we consider the class of M-Circuits $\mathcal{C}_{\left\{\mathbb{F}_{q_{1}}, \ldots, \mathbb{F}_{q_{f}}\right\}}^{\left(\left\{\mathbb{S}_{1}, \mathbb{S}_{s}, \emptyset\right)\right.}$. Later we 
shall remove this restriction, at the expense of introducing more rounds of communication.

Our construction extends Katz et al.'s construction [23] for arbitrary finite fields. Since we work with M-Circuits, we do not consider operations such as AND, multiplication or squaring (as in Katz et al.'s presentation), but rather we formalize the protocol in term of generic correlated randomness sources over arbitrary finite fields, along with calls to the reveal() function. As we have already explained, such a representation is universal, and can lead to optimizations (which we will discuss later).

We first describe the specific underlying MPC protocol to securely compute an M-Circuit instance that we will exploit in our MPCitH protocol. Then, we present an MPCitH-based HVZK argument of knowledge based on the input M-Circuit instance. Initially, we present a protocol which checks the correlated randomness sources using the cut-and-choose paradigm. This method works for arbitrary sources. In a latter section we present another methodology which works for some specific correlated randomness sources which is based on the sacrificing idea used in some actively secure MPC protocols.

\subsection{The Underlying MPC Protocol}

The MPCitH protocol we utilize will make use of a very simple (passively secure) MPC protocol based on full threshold secret sharing in the pre-processing model. The function $F$ we will be evaluating is assumed to have (some) sensitive input variables, but no sensitive output variables. The $N$ parties in the protocol we will denote by $P_{1}, \ldots, P_{N}$.

Offline Phase. We define an ideal functionality for the offline phase, which implements the generation of suitable correlated randomness according to the sources required by the M-Circuit. This is given in Figure 2 .

\section{MPC Offline Functionality $\mathcal{F}_{\text {Offline }}^{\mathcal{S}}$}

For every source $\mathbb{S} \in \mathcal{S}$ we define a command which operates as follows:

$\mathbb{S}: \quad$ On input of $(\mathbb{S})$ the functionality proceeds as follows

1. Generate $\left(v_{1}, \ldots, v_{t}\right)$ according to the source definition $\mathbb{S}$.

2. For all variables $v_{i}$ of type $\left(q_{i}, s\right)$ wait for shares $v_{i, j}$ from the adversary $\mathcal{A}$.

3. On receiving these shares complete them to a full set of shares $\sum_{j \notin \mathcal{A}} v_{i, j}=$ $v_{i}-\sum_{j \in \mathcal{A}} v_{i, j}$ and send the relevant $v_{i, j}$ to the honest parties.

4. Output $v_{i}$ when it is of type $\left(q_{i},-\right)$ to all parties.

Figure 2. Functionality $\mathcal{F}_{\text {Offline }}^{\mathcal{S}}$

Online Phase. We wish to implement the passively MPC/SFE functionality given in Figure 3, in which we assume the sensitive inputs are assigned to specific 
parties. This is done using the online phase given in Figure 4, which is defined in the $\mathcal{F}_{\text {Offline-hybrid model. }}$

\section{Passively Secure MPC/SFE Functionality $\mathcal{F}_{\mathrm{MPC}}$}

Given a function $F$ defined over finite fields with input variables $V_{I}$ and output variables $V_{O}$, the functionality proceeds as follows.

1. For each input variable $v \in V_{I}$ :

(a) If input variable $v$ of type $(q, s)$ is assigned to party $P_{i}$ then wait for party $P_{i}$ to enter the value $v$.

(b) If input variable $v$ is of type $(q,-)$ then wait for all parties to input the same value $v$.

2. Compute the function $F$ on $\left(v_{1}, \ldots, v_{t}\right)$ and output the output variables to all parties (recall we assume $F$ has no sensitive output variables).

Figure 3. Passively Secure MPC/SFE Functionality $\mathcal{F}_{\mathrm{MPC}}$

Security. We let $\mathcal{A}$ denote an adversary which statically corrupts a subset of the parties. We abuse notation slightly by referring to $\mathcal{A}$ both as the adversary, and as the set of parties which it has corrupted. We define $\operatorname{view}^{\left\{\mathcal{A}, \Pi_{\mathrm{MPC}}\right\}}(C)$ to be the view of $\mathcal{A}$ during the execution of the protocol $\Pi_{\text {MPC }}$ on the function $F$ represented by the M-Circuit $C$ in the $\mathcal{F}_{\text {Offline-hybrid model. This view consists }}$ of the inputs of the parties in $\mathcal{A}$, the shares of the correlated randomness the parties in $\mathcal{A}$ receive from $\left\{\mathbb{S}_{1}, \ldots, \mathbb{S}_{s}\right\}$, and the messages they obtain from the other parties while evaluating the protocol. The security of $\Pi$ is stated in the following theorem, the proof of which is given in the full version.

Theorem 4.1. For every subset of parties $\mathcal{A} \subseteq\left\{P_{1}, \cdots, P_{N}\right\}$, with $|\mathcal{A}| \leq N-$ 1 , there exists a probabilistic polynomial-time algorithm $\mathcal{S}$, with access to the functionality $\mathcal{F}_{\mathrm{MPC}}$, such that $\left\{\mathcal{S}\left(\mathcal{A}, F, v_{\mathcal{A}}\right)\right\} \equiv \operatorname{view}^{\left\{\mathcal{A}, \Pi_{M P C}\right\}}(C)$, where $v_{\mathcal{A}}$ are the function inputs of the parties in $\mathcal{A}$.

The equivalence relation is a perfect equivalence if the $M$-Circuit $C$ is a perfectly secure implementation of the functionality $F$, and is a statistical equivalence if the M-Circuit is a statistically secure implementation of F.

\subsection{Sub-Procedures for MPCitH}

In this subsection we collect together a number of sub-procedures and algorithms for our general MPCitH protocol for M-Circuits.

Pseudo-Random Generator. We let $\mathrm{PRG}_{q}$ denote a pseudo-random function, which on input of a key seed and an index $j$ outputs a (pseudo-) uniformly random element of the finite field $\mathbb{F}_{q}$. We let $\mathrm{PRF}_{\lambda}$ denote an equivalent function which outputs values in $\{0,1\}^{\lambda}$. 


\section{Passively Secure MPC/SFE Protocol $\Pi_{\mathrm{MPC}}^{(\mathcal{F}, \mathcal{S})}(C)$}

Given a function $F$ defined over finite fields with input variables $V_{I}$ and output variables $V_{O}$ represented as an M-Circuit $C$ in the class $\mathcal{C}_{\mathcal{F}}^{(\mathcal{S}, \emptyset)}$, the protocol proceeds as follows.

1. For each input variable $v \in V_{I}$

(a) If input variable $v$ of type $(q, s)$ is assigned to party $P_{i}$ then party $P_{i}$ shares $v=\sum v_{j}$ and sends $v_{j}$ to party $P_{j}$.

(b) If input variable $v$ is of type $(q,-)$ then all parties agree on the value $v$.

2. Now execute the M-Circuit line by line (as above).

- For a $x$.reveal() command, party $P_{i}$ sends his share $x_{i}$ to all parties.

- For a call to a correlated randomness source $\mathbb{S} \in \mathcal{S}$, make the appropriate call to the functionality $\mathcal{F}_{\text {Offline }}$.

- For an arithmetic operation, perform the associated operation on the linear secret sharing scheme given above.

3. Finally, for a terminate operation, for each variable $v \in V_{O}$ the parties output their (necessarily opened) value $v$ as their output.

Figure 4. Passively Secure MPC/SFE Protocol $\Pi_{\mathrm{MPC}}^{(\mathcal{F}, \mathcal{S})}(C)$

GenAux Function. To a correlated randomness source $\mathbb{S}$ we associate a deterministic algorithm GenAux ${ }^{\mathbb{S}}$ which on input of a given assignment to the variables $\left\{v_{1}, \ldots, v_{t}\right\}$ in the source will output a set of variables $\left\{v_{1}^{\prime}, \ldots, v_{t}^{\prime}\right\}$ of the same types. The output should satisfy the following equality of distributions, where $v_{1} \leftarrow \mathbb{F}_{p_{1}}$ means sample $v_{1}$ uniformly from the field $\mathbb{F}_{p_{1}}$,

$$
\begin{aligned}
&\left\{\left(v_{1}+v_{1}^{\prime}, \ldots, v_{t}+v_{t}^{\prime}\right):\right. v_{i} \leftarrow \mathbb{F}_{p_{i}} \text { for } i=1, \ldots, t \\
&\left.\left(v_{1}^{\prime}, \ldots, v_{t}^{\prime}\right) \leftarrow \operatorname{GenAux}^{\mathbb{S}}\left(v_{1}, \ldots, v_{t}\right)\right\} \\
& \equiv\left\{\left(v_{1}, \ldots, v_{t}\right):\left(v_{1}, \ldots, v_{t}\right) \leftarrow \mathbb{S}\right\}
\end{aligned}
$$

Note, there can be many ways for a given source to define the algorithm GenAux; some are more compact than others. For example take the source Triple which has (at least) the two following definitions for GenAux.

1. GenAux ${ }^{\text {Triple }}(a, b, c)=(0,0, a \cdot b-c)$.

2. GenAux ${ }^{\text {Triple }}(a, b, c)=(x-a, y-b, z-c)$ where $x, y$ are deterministically selected from $\mathbb{F}_{p}$ by GenAux Triple using a PRG with the seed $H(a, b, c)$, for some hash function $H$, and $z=x \cdot y$.

The first of these is more efficient in our application as the user knows the first two coordinates are always zero, and can therefore drop them from any data transferred. It turns out the first is also better for one of our optimizations we present later.

Sources which require some specific distribution, such as the Bit source from earlier, can be produced by defining $\operatorname{GenAux}^{\mathrm{Bit}}(a)=(a-b)$ where $b=H(a) \& 1$ for some hash function $H$. 
GenShares. To each correlated randomness source $\mathbb{S}$, with variables $\left\{v_{1}, \ldots, v_{t}\right\}$ we associate the following seeds:

1. If $v_{i}$ is of type $\left(q_{i},-\right)$ then we associate a single seed $\operatorname{seed}_{i}^{\mathbb{S}}$.

2. If $v_{i}$ is of type $\left(q_{i}, s\right)$ then we associate $N$ seeds $\operatorname{seed}_{i, j}^{\mathbb{S}}$ for $j=1, \ldots, N$.

We also associate a counter $\mathrm{cnt}^{\mathbb{S}}$, which on initialization of the source is set to zero. In the MPCitH protocol below when $\mathbb{S}$ is called we execute an algorithm GenShares ${ }^{\mathbb{S}}$ (given in Figure 5) which takes as input the above seeds and the counter $\mathrm{cnt}^{\mathbb{S}}$ and produces a sample from the randomness source presented as a sharing amongst the parties, as well as the correction term. We write $\left(\left\{v_{i}\right\}_{\dagger},\left\{v_{i, j}\right\}_{*}\right.$, aux, $\left.\mathrm{cnt}^{\mathbb{S}}\right) \leftarrow \operatorname{GenShares}^{\mathbb{S}}\left(\left\{\operatorname{seed}_{i}^{\mathbb{S}}\right\}_{\dagger},\left\{\operatorname{seed}_{i, j}^{\mathbb{S}}\right\}_{*}, \mathrm{cnt}^{\mathbb{S}}\right)$.

\section{The GenShares ${ }^{\mathbb{S}}$ Algorithm}

1. For all variables $v_{i}$ output by $\mathbb{S}$ of type $(q,-)$ execute

(a) $v_{i} \leftarrow \mathrm{PRG}_{q_{i}}\left(\right.$ seed $\left._{i}^{\mathbb{S}}, \mathrm{cnt}^{\mathbb{S}}\right)$

2. For all variables $v_{i}$ output by $\mathbb{S}$ of type $(q, s)$ execute

(a) $v_{i, j} \leftarrow \mathrm{PRG}_{q_{i}}\left(\operatorname{seed}_{i, j}^{\mathbb{S}}, \mathrm{cnt}^{\mathbb{S}}\right)$ for $j=1, \ldots, N$.

(b) $v_{i} \leftarrow \sum_{j=1}^{n} v_{i, j}$ for all $i \in 1, \ldots, t$.

3. $\mathrm{cnt}^{\mathbb{S}} \leftarrow \mathrm{cnt}^{\mathbb{S}}+1$.

4. aux $=\left(v_{1}^{\prime}, \ldots, v_{t}^{\prime}\right) \leftarrow \operatorname{GenAux}^{\mathbb{S}}\left(v_{1}, \ldots, v_{t}\right)$.

5. $v_{i} \leftarrow v_{i}+v_{i}^{\prime}$ for all $i$.

6. $v_{i, N} \leftarrow v_{i, N}+v_{i}^{\prime}$ for all $i$ such that $v_{i}$ has type $\left(q_{i}, s\right)$.

7. Output $\left(\left\{v_{i}\right\}_{\dagger},\left\{v_{i, j}\right\}_{*}\right.$, aux, $\left.\mathrm{cnt}^{\mathbb{S}}\right)$, where $\dagger$ denotes $v_{i}$ has type $\left(q_{i},-\right)$ and $*$ denotes $v_{i}$ has type $\left(q_{i}, s\right)$.

Figure 5. The GenShares ${ }^{\mathbb{S}}$ algorithm for a source $\mathbb{S}$

\subsection{The Construction of HVZK Argument of Knowledge}

We can now present our generalization of the MPCitH protocols of $[5,23]$ to the case of arbitrary M-Circuits. Our initial construction uses the cut-and-choose checking paradigm, but later we will also consider the other checking approach, i.e. sacrificing, that we show to be more efficient for particular cases. Recall at this point we assume an M-Circuit $C$ is given in the class $\mathcal{C}_{\left\{\mathbb{F}_{q_{1}}, \ldots, \mathbb{F}_{q_{f}}\right\}}^{\left(\left\{\mathbb{S}_{1}, \ldots \mathbb{S}_{s}, \emptyset\right)\right.}$. The MCircuit has no sensitive output variables, but there are a set of sensitive input variables, which we denote by $\mathbf{w}$. The prover wishes to show that he knows a witness for these output variables, which by abuse of notation we also call w, such that the M-Circuit produces a given output.

At a high level the proof proceeds by the prover simulating the $N$-party MPC protocol from Section 4.1 in his head and executes it over an additive sharing of $\mathbf{w}$, along with calls to the correlated randomness sources $\left(\left\{\mathbb{S}_{1}, \ldots, \mathbb{S}_{s}\right\}, \emptyset\right)$, which are performed using the algorithm GenShares ${ }^{\mathbb{S}}$ given above. Clearly, as the secret sharing scheme is executed in prover's head, the prover might try to cheat and 
convince the verifier about a false statement. To prevent such issues, and hence to obtain a negligible soundness error, the construction allows the verifier to challenge the prover. Namely, at the end of the first round the prover commits (by the above commitment scheme) to the views of $N$ parties in $M$ executions. Then, the verifier (randomly) challenges a subset of executions $E \subset[M]$ of size $\tau$ for which all correction terms induced by calls to the correlated randomness sources will be revealed and verified. The verifier also (randomly) challenges a single party $j \in[N]$, such that all parties views are opened to him (bar party $j$ ) for all $e \in[M] \backslash E$.

After revealing the secret information for the challenged executions and parties, e.g. the master seeds, the challenged parties' seeds, or the commitments, the verifier recomputes (either using directly the values sent by the Prover, or by using the parties' seeds and correction terms to emulate the secret sharing scheme) and checks the commitments and final output of the M-Circuit.

In the described HVZK argument, intuitively, zero-knowledge is achieved relying on the fact that the M-Circuit is secure (its trace is simulatable) and the revealed data are only random values which are independent of the witness $\mathbf{w}$. Thus the $N-1$ views that are revealed do not reveal anything as the underlying MPC protocol is passively secure against $N-1$ semi-honest parties.

In our protocol description, as before, we use $\langle x\rangle_{q}$ to denote a sensitive variable (associated to an additive sharing $x=\sum_{j \in[N]} x_{i}$ ) and $\{x\}_{q}$ to denote a non-sensitive variable within the M-Circuit. As input to both the protocol and the verifier we have a general M-circuit $C$ in the class $\mathcal{C}_{\mathcal{F}}^{(\mathcal{S}, \emptyset)}$, with the set of finite fields $\mathcal{F}=\left\{\mathbb{F}_{q_{1}}, \ldots, \mathbb{F}_{q_{f}}\right\}$, a set of Correlated Randomness Sources $\mathcal{S}=\left\{\mathbb{S}_{1}, \ldots, \mathbb{S}_{s}\right\}$, and no gadget. We assume that the prover and verifier have agreed on the non-sensitive input variables to the M-Circuit, and the prover additionally has an assignment to the sensitive input variables (witness) such that the M-Circuit evaluates to a given public output. Due to the similarity with the protocol from [23] we present the specific protocols in the full version.

\section{$5 \quad$ Using Different Correlated Randomness Sources}

In the case of MPC protocols if one wants to add a new form of correlated randomness to a protocol then this equates to a more complex and costly offline phase. When using our cut-and-choose methodology for checking the correlated randomness sources in the MPCitH protocol we have already paid the cost of introducing a single source. Thus introducing new sources is essentially 'for free', and can indeed reduce complexity of this stage by requiring less data to check, as well as reducing proof complexity (both in time to produce/verify and in terms of size). Thus a new correlated randomness source should aim to reduce the online cost $c_{\text {online }}$, whilst not increasing $c_{\text {offline }}$ (and the associated size of the auxiliary data needed for the resource) by a similar amount. We give two examples, one arithmetic and one non-arithmetic, 


\subsection{Dot-Product Computation}

As an example, suppose in an M-Circuit program one is given sensitive vectors $\langle\mathbf{x}\rangle_{q}$ and $\langle\mathbf{y}\rangle_{q}$ of size $k$ and one wishes to compute their dot-product. The naive way of doing the dot product would be to call the correlated randomness source for triple generation $k$ times; thus receiving $\left\{\left(\left\langle a_{i}\right\rangle_{q},\left\langle b_{i}\right\rangle_{q},\left\langle c_{i}\right)\right\rangle_{q}\right\}_{i \in[k]}$, and then doing the Beaver multiplication trick $k$ times.

$$
\begin{aligned}
& \left\langle z_{1}\right\rangle_{q}=\left\langle c_{1}\right\rangle_{q}-\left(x_{1}-a_{1}\right) \cdot\left\langle b_{1}\right\rangle_{q}-\left(y_{1}-b_{1}\right) \cdot\left\langle a_{1}\right\rangle_{q}+\left(x_{1}-a_{1}\right) \cdot\left(y_{1}-b_{1}\right) \\
& \quad \ldots \ldots \\
& \left\langle z_{k}\right\rangle_{q}=\left\langle c_{k}\right\rangle-\left(x_{k}-a_{k}\right) \cdot\left\langle b_{k}\right\rangle_{q}-\left(y_{k}-b_{k}\right) \cdot\left\langle a_{k}\right\rangle_{q}+\left(x_{k}-a_{k}\right) \cdot\left(y_{k}-b_{k}\right)
\end{aligned}
$$

Finally we obtain $\langle z\rangle_{q}=\sum_{i \in[k]}\left\langle z_{i}\right\rangle_{q}$. The $k$ calls to the correlated randomness source, however, require $k$ correction terms to make sure that $\left\langle c_{i}\right\rangle=\left\langle a_{i} \cdot b_{i}\right\rangle$ for all $i$. Thus these $k$ terms need to be added to the proof. However, by introducing a different correlated randomness source tailored to this specific operation we can replace these $k$ correction terms with a single term. To see this note that we could also write

$$
\langle z\rangle_{q}=\sum\left\langle c_{i}\right\rangle_{q}-\sum\left(\left(x_{i}-a_{i}\right) \cdot\left\langle b_{i}\right\rangle_{q}-\left(y_{i}-b_{i}\right) \cdot\left\langle a_{i}\right\rangle_{q}+\left(x_{i}-a_{i}\right) \cdot\left(y_{i}-b_{i}\right)\right) .
$$

Therefore, the necessary pre-processing data could be obtained by defining a new correlated source which produces values of the form

$$
\left\langle a_{1}\right\rangle_{q}, \ldots,\left\langle a_{k}\right\rangle_{q},\left\langle b_{1}\right\rangle_{q}, \ldots,\left\langle b_{k}\right\rangle_{q},\langle c\rangle_{q} \text { where } c=\sum_{i \in[k]} c_{i}=\sum_{i \in[k]} a_{i} \cdot b_{i} .
$$

Using this source we thus need only one correction term for $c$, thus saving ( $M-$ $\tau) \cdot(k-1)$ field elements of communication for the pre-processing material when using our cut-and-choose method for source correctness verification.

\subsection{Matrix triples}

This is a trick known in the MPC literature that can be easily applied to the setting of MPCitH. Consider $\langle\mathbf{X}\rangle_{q}$ and $\langle\mathbf{Y}\rangle_{q}$ two matrices of size $n \cdot m$ and $m \cdot l$ respectively. The naive way of computing $\langle\mathbf{Z}\rangle_{q}=\langle\mathbf{X} \cdot \mathbf{Y}\rangle_{q}$ requires $O(n \cdot m \cdot l)$ calls to the correlated randomness source for triple generation. However if one has access to a correlated randomness source for matrix triples $\left(\langle\mathbf{A}\rangle_{q},\langle\mathbf{B}\rangle_{q},\langle\mathbf{C}\rangle_{q}\right)$ such that $\mathbf{C}=\mathbf{A} \cdot \mathbf{B}$ and $\mathbf{A}, \mathbf{B}$ are two matrices of size $n \cdot m$ and $m \cdot l$ respectively, one can perform the matrix multiplication much more efficiently. Indeed, this source only requires $n \cdot l$ auxiliary information and the multiplication protocol is similar to the classic Beaver multiplication, thus requires to reveal one $n \cdot m$ and one $m \cdot l$ matrix. 


\subsection{Tiny-Tables}

Interesting optimizations from the MPC world can be carried over directly to the MPCitH worlds using our abstraction. Consider for example the Tiny-Tables optimization, see [19] as extended by [25]. Suppose we wish, at some point in the computation, to compute a function $y=G(x)$ where $x, y \in \mathbb{F}_{q}$, for prime $q$, and $x$ is known to be restricted to come from a small domain $\mathcal{D} \subset \mathbb{F}_{q}$ of size $d-1$, with $d<q / 2$. For simplicity assume $\mathcal{D}=\{0, \ldots, d-1\}$ in what follows.

We can define the correlated randomness source $\mathbb{S}_{G}$ which outputs sensitive values $\langle s\rangle,\left\langle g_{0}\right\rangle, \ldots,\left\langle g_{d-1}\right\rangle$, subject to the constraints that $s$ is uniformly randomly chosen from $\mathbb{F}_{q}$ and that

$$
g_{i}=G\left(\begin{array}{ll}
(s+i \quad(\bmod q)) & (\bmod d)
\end{array}\right) .
$$

Evaluation of the table on a shared value $\langle x\rangle$, whose value is guaranteed to lie in $\mathcal{D}$, can then be performed by opening the value $\langle h\rangle=\langle x\rangle-\langle s\rangle$, to obtain $h$ (which we reduce into the centred interval $(-q / 2, \ldots, q / 2)$ ) and then taking the result of the table look up as $\left\langle g_{h}(\bmod d)\right\rangle$.

In the case of MPCitH the size of the output of GenAux will depend on the input domain size $d$. Thus the Tiny-Table approach will result in a smaller proof if the table size is less than the multiplicative complexity of the function $G$, assuming the only alternative is to compute $G$ via an arithmetic circuit.

\section{Sacrificing}

Another trick we can take from the MPC world and apply to the world of MPCitH protocols, directly from our M-Circuit definition, is that of sacrificing. At a high level one can consider the cut-and-choose component of the MPCitH protocol i.e. where the verifier selects the set $E$ and the prover opens all the correlated randomness from the executions in $E$, as a method to turn a passively secure offline phase into an actively secure offline phase for the underlying MPC protocol. The method of cut-and-choose is very general, and thus applies to any correlated randomness source. However, some correlated randomness sources are arithmetic in nature and thus can be checked using arithmetic means. This is well known in the MPC literature, and is called sacrificing. We note a similar trick was proposed in [5] but not in the generality we present.

We refer to correlated randomness sources for which one can execute a method akin to sacrificing as a Checkable Correlated Randomness Source. Using such a check, as opposed to the generic cut-and-choose methodology from earlier, can introduce efficiencies. However, it comes at the cost of needing a five-round, as opposed to a three-round protocol.

The basic idea is to modify the correlated randomness source so that it produces an additional correlation, which is 'sacrificed' so as to check the correctness of the desired correlation. The correctness check makes use of a verifier defined random nonce, which can be fixed across all of the checks. To simplify our presentation we consider the case where all the variables in a randomness source 
are defined over the same finite field $\mathbb{F}_{q}$, where $q$ is large; extending to smaller and different finite fields is trivial.

In terms of an M-Circuit definition, suppose we have an initial M-Circuit utilizing a desired source $\mathbb{S}$ which produces correlated variables x. However, to compile it we utilize a related source $\mathbb{S}^{\prime}$ whose output variables are of the form $(\mathbf{x}, \mathbf{y})$ and which has a function generating auxiliary input GenAux ${ }^{\mathbb{S}^{\prime}}$. There

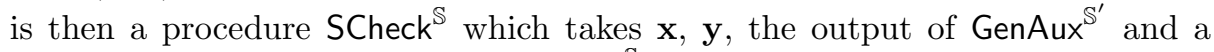
challenge value $t$. The procedure $\mathrm{SCheck}^{\mathbb{S}}$ which outputs a single bit $b$; if $b=0$ then the value $\mathbf{x}$ is not from the same distribution as $\mathbb{S}$ and if $b=1$ then it is. The probability that the bit $b$ is incorrect is bounded by a value $\epsilon^{\mathbb{S}^{\prime}}$, with the probability being a function of the choice of the challenge value $t$. We say that such a source $\mathbb{S}^{\prime}$ is a Checkable Correlated Randomness Source. The required modifications to our MPCitH protocol we give in the full version. Our protocol is similar to the one before, except now the prover cannot commit to the views of the M-Circuit evaluation until it knows the versifier's choice for $\mathbf{t}^{\mathbb{S}^{\prime}}$ for every $\mathbb{S} \in \mathcal{S}_{\text {check. }}$. On the other hand it must commit to the seeds generating the players secret sharings before it knows the value of $\mathbf{t}^{\mathbb{S}^{\prime}}$. This introduces an extra two rounds of communication, resulting in a total of five rounds of interaction.

We note that to determine how many calls we make to the random sources, we need to know the value of all the variables in the M-circuit. Therefore, the output of all sources (checkable and non-checkable) must be known before receiving the cut-and-choose challenge. An attentive reader may thus point out that if one has to pay the soundness cost of cut-and-choose, then there is no practical reason for adding sacrificing on top of it, as all correlated randomness sources that can be checked by sacrificing can also be checked by cut-and-choose. This is indeed the case, and in the case of an M-Circuit $C \in \mathcal{C}_{\mathcal{F}}^{(\mathcal{S}, \emptyset)}$, one should, in practice, use the sacrificing technique only if $\forall \mathbb{S} \in \mathcal{S}, S \in \mathcal{S}_{\text {check }}$, and completely ignore the cut-and-choose part of the protocol ( $\operatorname{set} \tau=0$ ).

However the usefulness of our 5-round protocol will be clear once we describe gadgets. Indeed, gadgets will be treated in a similar way as checkable correlated randomness sources, with the exception that, unlike correlated randomness sources, the cut-and-choose technique can not be used to check the correctness of their execution.

Example Checkable Correlated Randomness Sources: Note, the "program' for the check SCheck ${ }^{\mathbb{S}}$ can be expressed as an M-Circuit in the class $\mathcal{C}_{\mathbb{F}_{q}}^{(\emptyset, \emptyset)}$. Thus property of being a checkable correlated randomness source is a function not only of the procedure $\mathrm{SCheck}^{\mathbb{S}}$ existing, but also of the definition of the function GenAux ${ }^{\mathbb{S}^{\prime}}$ as we now illustrate.

Triple. Recall the source Triple produces tuples $(\langle a\rangle,\langle b\rangle,\langle c\rangle)$ such that $c=a \cdot b$. Our 'extended' source Triple' produces values $(\langle a\rangle,\langle b\rangle,\langle c\rangle,\langle d\rangle,\langle e\rangle)$ where it is 'claimed' that $c=a \cdot b$ and $e=b \cdot d$. This validity can be verified by the following algorithm which takes as input a public value $t \in \mathbb{F}_{q}$ (which can be the same for every output of Triple'). 
1. $\langle\rho\rangle \leftarrow t \cdot\langle a\rangle-\langle d\rangle$.

2. $\rho \leftarrow\langle\rho\rangle$.reveal () .

3. $\langle r\rangle \leftarrow t \cdot\langle c\rangle-\langle e\rangle-\rho \cdot\langle b\rangle$.

4. $r \leftarrow\langle r\rangle \cdot$ reveal () .

5. Reject if $r \neq 0$.

Note, this algorithm is an M-Circuit in the class $\mathcal{C}_{\mathbb{F}_{q}}^{(\emptyset, \emptyset)}$. Also note that the algorithm reveals no information about the values $\langle a\rangle,\langle b\rangle$ and $\langle c\rangle$. Also note, that for a valid tuple we have $r=t \cdot c-e-\rho \cdot b=t \cdot a \cdot b-b \cdot d-(t \cdot a-d) \cdot b=0$, and note that if $c \neq a \cdot b$ and $e \neq b \cdot d$ then we have $r=t \cdot(c-a \cdot b)+e+b \cdot d$ which will equal zero with probability $\epsilon^{\text {Triple }^{\prime}}=1 / q$, when $t$ is chosen independently of the output of Triple'.

However, whilst this verifies that the $(\langle a\rangle,\langle b\rangle,\langle c\rangle)$ variables output by Triple' satisfy the desired multiplicative relationship, it does not on its own demonstrate that the distribution of $(\langle a\rangle,\langle b\rangle,\langle c\rangle)$ is correct; namely that $\langle a\rangle$ and $\langle b\rangle$ are chosen uniformly at random. To ensure this we need to examine how GenAux ${ }^{\text {Triple }^{\prime}}$ is defined. Mirroring our two previous instantiations of GenAux ${ }^{\text {Triple }}$ we have

1. GenAux Triple' $^{\prime}(a, b, c, d, e)=(0,0, a \cdot b-c, b \cdot d-e)$.

2. GenAux ${ }^{\text {Triple }^{\prime}}(a, b, c, d, e)=(x-a, y-b, z-c, u-d, w-e)$ where $x, y, u$ are deterministically selected from $\mathbb{F}_{p}$ by GenAux ${ }^{\text {Triple' }}$ using a PRG with the seed $H(a, b, c, d, e)$, for some hash function $H, z=x \cdot y$ and $w=y \cdot u$.

The first case produces the correct distribution irrespective of what the prover computes, whereas the second case does not. In the second case a cheating prover can deviate from the protocol and make $\langle a\rangle$ follow any distribution they desire.

Bit. As remarked earlier this is the more interesting correlated randomness source in applications, as it enables far more efficient M-Circuit representations of functions. The source Bit produces a value $\langle b\rangle$ such that $b$ is guaranteed to lie in $\{0,1\}$. However, whilst in an MPC protocol there is a sacrificing methodology for Bits, this does not translate over to the MPCitH paradigm as one needs a way of verifying the bits are uniformly selected. Thus checking the source Bit seems to require cut-and-choose.

\section{Executable Gadgets}

Up until now we have considered for our MPCitH protocols only M-Circuits from classes of the form $\mathcal{C}_{\mathcal{F}}^{(\mathcal{S}, \emptyset)}$, i.e. M-Circuits with no gadgets. A gadget captures an essential non-linear subroutine within an M-Circuit. By abstracting it away, we simplify the composition of special-purpose protocols for such subroutines within a more generic M-Circuit. Whilst M-Circuits can describe arbitrary gadgets, only special gadgets, which we call executable gadgets are able to be supported by the MPCitH protocol.

We proceed to the formal definition of an executable gadget, which we define over a single finite field $\mathbb{F}_{q}$ of large characteristic for ease of exposition, and then we present two examples of executable gadgets for MPCitH protocols. 
Definition 7.1 (Executable Gadget). An Executable Gadget $\mathbb{G}$ is an object defined by

I. A function $G$ with (possibly zero) inputs and (at least one) output in $\mathcal{F}$.

II. A GenAux ${ }^{\mathbb{G}}$ function that fixes the auxiliary information needed to correct a uniformly random $\mathbf{y}$ to be equal to $G(\mathbf{x})$, i.e. $\operatorname{GenAux}{ }^{\mathbb{G}}(\mathbf{x}, \mathbf{y})=G(\mathbf{x})-\mathbf{y}$.

III. A GCheck ${ }^{\mathbb{G}} M$-Circuit in the class $\mathcal{C}_{\mathcal{F}}^{(\mathcal{S}, \emptyset)}$ for a set of randomness sources $\mathcal{S}$, the function which takes as input $\mathbf{x}, \mathbf{y}$, the output of $\operatorname{GenAux}^{\mathbb{G}}(\mathbf{x}, \mathbf{y})$ and a challenge value $t \in \mathcal{F}$. The procedure $\mathrm{GCheck}^{\mathbb{G}}$ which outputs a single bit $b$; if $b=0$ then the the values are inconsistent, i.e. the purported value of aux is not correct, and if $b=1$ then it is correct. The probability that the bit $b$ is incorrect is bounded by a value $\epsilon^{\mathbb{G}^{\prime}}$, with the probability being a function of the choice of the challenge value $t$.

Thus an executable gadget is very similar to the checkable randomness sources from the previous section. To process the gadget with in the MPCitH protocol we thus proceed just as we did for checkable randomness sources; the initial MCircuit $C$ is extended to an augmented circuit $C^{\prime}$ which includes all the necessary GCheck $^{\mathbb{G}}$ operations. As GCheck ${ }^{\mathbb{G}}$ itself potentially requires access to correlated randomness sources this might require the addition of addition correlated randomness source. Then the five round protocol is executed, so that the augmented circuit $C^{\prime}$ can be created (as it depends on the versifier's selection of the challenges $t$ in $\mathrm{GCheck}^{\mathbb{G}}$ ). The modification to the soundness error is the same as that introduced for checkable randomness sources.

The BitDecomp Gadget: The executable gadget BitDecomp for a given sensitive value $\langle x\rangle_{q}$ produces the $\left\lceil\log _{2}(q)\right\rceil$ sensitive values $\left\langle b_{i}\right\rangle_{q}$ such that $b_{i} \in\{0,1\}$ and $x=\sum_{i=0}^{\left\lceil\log _{2}(q)\right\rceil-1} b_{i} \cdot 2^{i}$. A simple example is the bit decomposition operation $\left(\left\langle b_{0}\right\rangle_{q}, \ldots,\left\langle b_{\left\lceil\log _{2}(q)\right\rceil-1}\right\rangle_{q} \leftarrow \operatorname{BitDecomp}(\langle x\rangle)\right.$, where $\forall i, b_{i} \in\{0,1\}$ and $\sum_{i} b_{i} 2^{i}=x$. See Figure 6 for a formal specification, note this checking procedure requires no random input from the verifier; this is because there is implicitly random input needed to check the (checkable) correlated randomness source Square which it requires.

In the above instantiation of BitDecomp we assumed that our randomness source Square was already checked by either cut-and-choose or sacrificing. However, we can obtain a further efficiency if we merge the checking of the output of Square with the checking of this bits produced in the gadget. To present this we give utilize a correlated randomness source USquare, which represents an unchecked square tuple. Namely, we check the output is correct neither by the sacrificing style check or via cut-and-choose. This allows us to present an more efficient check of the BitDecomp Gadget in Figure 7, where now we require the verifier to provide a random challenge $t \in \mathbb{F}_{q}$. At first sight it seems to involve the same number of reveals operations, but we actually save operations as we no longer need reveals to check the output of Square. 


\section{The BitDecomp Gadget}

I. Function $G:\left(\langle x\rangle_{q}\right) \mapsto\left(\left\langle b_{0}\right\rangle_{q}, \ldots,\left\langle b_{\left\lceil\log _{2}(q)\right\rceil-1}\right\rangle_{q}\right)$ such that $b_{i} \in\{0,1\}$ and $x=\sum_{i} b_{i} \cdot 2^{i}$

II. $\operatorname{GenAux}^{\operatorname{BitDecomp}}\left(x,\left(y_{0}, \ldots, y_{\lceil\log (q)\rceil-1}\right)\right)=\left(0, b_{0}-y_{0}, \ldots, b_{\left\lceil\log _{2}(q)\right\rceil-1}-\right.$ $y_{\left.\left\lceil\log _{2}(q)\right\rceil-1\right)}$

III. GCheck ${ }^{\text {BitDecomp }} \in \mathcal{C}_{\mathbb{F}_{q}}^{(\text {Square, } \emptyset)}$ : On input of $\left\langle b_{0}\right\rangle_{q}, \ldots,\left\langle b_{\left\lceil\log _{2}(q)\right\rceil-1}\right\rangle_{q},\langle x\rangle_{q}$ :

(a) flag $\leftarrow 1,\langle s\rangle_{q} \leftarrow 0$

(b) For $i$ from $\left\lceil\log _{2}(q)\right\rceil-1$ to 0 do

- $\langle a\rangle_{q},\left\langle a^{2}\right\rangle_{q} \leftarrow$ Square

- $\langle\alpha\rangle_{q} \leftarrow\left\langle b_{i}\right\rangle_{q}-\langle a\rangle_{q}$

- $\{\alpha\}_{q} \leftarrow\langle\alpha\rangle_{q} \cdot$ reveal ()

$-\langle r\rangle_{q} \leftarrow\{\alpha\}_{q} \cdot\left(\left\langle b_{i}\right\rangle_{q}+\langle a\rangle_{q}\right)+\left\langle a^{2}\right\rangle_{q}-\left\langle b_{i}\right\rangle_{q}$

- $\{r\}_{q} \leftarrow\langle r\rangle_{q} \cdot$ reveal ()

- If $\{r\}_{q} \neq 0$ then flag $\leftarrow 0$

- $\langle s\rangle_{q} \leftarrow 2 \cdot\langle s\rangle_{q}+\left\langle b_{i}\right\rangle_{q}$

(c) $\langle s\rangle_{q} \leftarrow\langle s\rangle_{q}-\langle x\rangle_{q}$

(d) $\{s\}_{q} \leftarrow\langle s\rangle_{q} \cdot \operatorname{reveal}()$

(e) If $\{s\}_{q} \neq 0$ then flag $\leftarrow 0$

(f) Return flag

Figure 6. The BitDecomp Gadget

\section{The Optimized BitDecomp Gadget}

I. Function $G:\left(\langle x\rangle_{q}\right) \mapsto\left(\left\langle b_{0}\right\rangle_{q}, \ldots,\left\langle b_{\left\lceil\log _{2}(q)\right\rceil-1}\right\rangle_{q}\right)$ such that $b_{i} \in\{0,1\}$ and $x=\sum_{i} b_{i} \cdot 2^{i}$

II. $\operatorname{GenAux}{ }^{\operatorname{BitDecomp}}\left(x,\left(y_{0}, \ldots, y_{\lceil\log (q)\rceil-1}\right)\right)=\left(0, b_{0}-y_{0}, \ldots, b_{\left\lceil\log _{2}(q)\right\rceil-1}-\right.$ $y_{\left.\left\lceil\log _{2}(q)\right\rceil-1\right)}$

III. GCheck ${ }^{\text {BitDecomp }} \in \mathcal{C}_{\mathbb{F}_{q}}^{(\text {Square, },)}$ : On input of $\left\langle b_{0}\right\rangle_{q}, \ldots,\left\langle b_{\left\lceil\log _{2}(q)\right\rceil-1}\right\rangle_{q},\langle x\rangle_{q}$ and a value $t$ from the verifier:

(a) flag $\leftarrow 1,\langle s\rangle_{q} \leftarrow 0$

(b) For $i$ from $\left\lceil\log _{2}(q)\right\rceil-1$ to 0 do

- $\langle a\rangle_{q},\left\langle a^{2}\right\rangle_{q} \leftarrow$ USquare

- $\langle\rho\rangle_{q} \leftarrow\left\langle b_{i}\right\rangle_{q}-t \cdot\langle a\rangle_{q}$

- $\{\rho\}_{q} \leftarrow\langle\rho\rangle_{q} \cdot$ reveal ()

- $\langle r\rangle_{q} \leftarrow\left\langle b_{i}\right\rangle_{q}-\{\rho\}_{q} \cdot\left(\left\langle b_{i}\right\rangle_{q}+t \cdot\langle a\rangle_{q}\right)-t^{2} \cdot\left\langle a^{2}\right\rangle_{q}$.

- $\{r\}_{q} \leftarrow\langle r\rangle_{q}$.reveal ()

- If $\{r\}_{q} \neq 0$ then flag $\leftarrow 0$

$-\langle s\rangle_{q} \leftarrow 2 \cdot\langle s\rangle_{q}+\left\langle b_{i}\right\rangle_{q}$

(c) $\langle s\rangle_{q} \leftarrow\langle s\rangle_{q}-\langle x\rangle_{q}$

(d) $\{s\}_{q} \leftarrow\langle s\rangle_{q} \cdot$ reveal()

(e) If $\{s\}_{q} \neq 0$ then flag $\leftarrow 0$

(f) Return flag

Figure 7. The Optimized BitDecomp Gadget 
The RNSDecomp Gadget: If we extend our definitions to rings $\mathbb{Z}_{q}$ with $q=$ $\prod_{i=1}^{k} p_{i}$ and $p_{i}$ primes, an interesting technique which has been widely used in cryptography is to make use of the Chinese Remainder Theorem. In MPCitH it is very easy for the prover to inject the residues of a sensitive variable such that the M-Circuit can operate on those residues. Since CRT reconstruction is a linear operation, it is also trivial to design a GCheck M-Circuit, as it suffice to apply the linear CRT reconstruction algorithm to the residues, and compare the result with the original value. An application of such a technique would then be to use the Tiny-Tables optimization described previously, but for functions with domain $\mathbb{Z}_{q}$ that can be computed residue-wise. By following the blueprint of [4], one would then create a table of the desired function for all the residues, thus going from a prohibitive size $q$ table to $k$ tables of total size $\sum p_{i}$. (e.g. exponentiation of a sensitive variable by a non-sensitive variable)

\section{The RNSDecomp get}

I. Function $G:\left(\langle x\rangle_{q}\right) \mapsto\left(\left\langle x_{1}\right\rangle_{p_{1}}, \ldots,\left\langle x_{k}\right\rangle_{p_{k}}\right)$ such that $x_{i} \in \mathbb{F}_{p_{i}}$ and $x=$ $\mathrm{CRT}\left(\left[x_{1}, \ldots, k_{k}\right],\left[p_{1}, \ldots, p_{k}\right]\right)$

II. GenAux ${ }^{\text {RNSDecomp }}\left(x,\left(y_{1}, \ldots, y_{k}\right)\right)=\left(0, x_{1}-y_{1}, \ldots, b_{k}-y_{k}\right)$

III. GCheck ${ }^{\text {RNSDecomp }} \in \mathcal{C}_{\mathbb{Z}_{q}, \mathbb{F}_{p_{1}}, \ldots, \mathbb{F}_{p_{k}}}^{(\emptyset, \emptyset)}$ : On input of $\left\langle x_{1}\right\rangle_{p_{1}}, \ldots,\left\langle x_{k}\right\rangle_{p_{k}},\langle x\rangle_{q}$ :
(a) flag $\leftarrow 1$
(b) $\langle s\rangle_{q} \leftarrow \operatorname{CRT}\left(\left[\left\langle x_{1}\right\rangle_{p_{1}}, \ldots,\left\langle x_{k}\right\rangle_{p_{k}}\right],\left[p_{1}, \ldots, p_{k}\right]\right)$ (Local operation)
(c) $\langle s\rangle_{q} \leftarrow\langle s\rangle_{q}-\langle x\rangle_{q}$
(d) $\{s\}_{q} \leftarrow\langle s\rangle_{q} \cdot \operatorname{reveal}()$
(e) If $\{s\}_{q} \neq 0$ then flag $\leftarrow 0$
(f) Return flag

Figure 8. Residue Number System Decomposition

\section{Acknowledgments}

This work has been supported in part by ERC Advanced Grant ERC-2015-AdGIMPaCT, by the Defense Advanced Research Projects Agency (DARPA) and Space and Naval Warfare Systems Center, Pacific (SSC Pacific) under contract No. HR001120C0085 and FA8750-19-C-0502, by the FWO under an Odysseus project GOH9718N, and by CyberSecurity Research Flanders with reference number VR20192203.

\section{References}

1. Aly, A., Cong, K., Cozzo, D., Keller, M., Orsini, E., Rotaru, D., Scherer, O., Scholl, P., Smart, N.P., Tanguyu, T., Wood, T.: SCALE and MAMBA documentation, v1.11 (2021), https://homes.esat.kuleuven.be/ nsmart/SCALE/ Documentation.pdf 
2. Aly, A., Orsini, E., Rotaru, D., Smart, N.P., Wood, T.: Zaphod: Efficiently combining LSSS and garbled circuits in SCALE. In: Brenner, M., Lepoint, T., Rohloff, K. (eds.) Proceedings of the 7th ACM Workshop on Encrypted Computing \& Applied Homomorphic Cryptography, WAHC@CCS 2019, London, UK, November 11-15, 2019. pp. 33-44. ACM (2019), https://doi.org/10.1145/3338469.3358943

3. Ames, S., Hazay, C., Ishai, Y., Venkitasubramaniam, M.: Ligero: Lightweight sublinear arguments without a trusted setup. In: Thuraisingham, B.M., Evans, D., Malkin, T., Xu, D. (eds.) ACM CCS 2017. pp. 2087-2104. ACM Press (Oct / Nov 2017)

4. Ball, M., Malkin, T., Rosulek, M.: Garbling gadgets for Boolean and arithmetic circuits. In: Weippl, E.R., Katzenbeisser, S., Kruegel, C., Myers, A.C., Halevi, S. (eds.) ACM CCS 2016. pp. 565-577. ACM Press (Oct 2016)

5. Baum, C., Nof, A.: Concretely-efficient zero-knowledge arguments for arithmetic circuits and their application to lattice-based cryptography. In: Kiayias, A., Kohlweiss, M., Wallden, P., Zikas, V. (eds.) PKC 2020, Part I. LNCS, vol. 12110 , pp. 495-526. Springer, Heidelberg (May 2020)

6. Beaver, D.: Efficient multiparty protocols using circuit randomization. In: Feigenbaum, J. (ed.) CRYPTO'91. LNCS, vol. 576, pp. 420-432. Springer, Heidelberg (Aug 1992)

7. Bell, D.E., LaPadula, L.J.: Secure computer systems: Mathematical foundations. MITRE Corporation Technical Report 2547 (1973)

8. Ben-Or, M., Goldwasser, S., Wigderson, A.: Completeness theorems for noncryptographic fault-tolerant distributed computation (extended abstract). In: 20th ACM STOC. pp. 1-10. ACM Press (May 1988)

9. Bhadauria, R., Fang, Z., Hazay, C., Venkitasubramaniam, M., Xie, T., Zhang, Y.: Ligero++: A new optimized sublinear IOP. In: Ligatti, J., Ou, X., Katz, J., Vigna, G. (eds.) ACM CCS 20. pp. 2025-2038. ACM Press (Nov 2020)

10. Catrina, O., de Hoogh, S.: Improved primitives for secure multiparty integer computation. In: Garay, J.A., Prisco, R.D. (eds.) SCN 10. LNCS, vol. 6280, pp. 182 199. Springer, Heidelberg (Sep 2010)

11. Catrina, O., Saxena, A.: Secure computation with fixed-point numbers. In: Sion, R. (ed.) FC 2010. LNCS, vol. 6052, pp. 35-50. Springer, Heidelberg (Jan 2010)

12. Chase, M., Derler, D., Goldfeder, S., Orlandi, C., Ramacher, S., Rechberger, C., Slamanig, D., Zaverucha, G.: Post-quantum zero-knowledge and signatures from symmetric-key primitives. In: Thuraisingham, B.M., Evans, D., Malkin, T., Xu, D. (eds.) ACM CCS 2017. pp. 1825-1842. ACM Press (Oct / Nov 2017)

13. Chaum, D., Crépeau, C., Damgård, I.: Multiparty unconditionally secure protocols (extended abstract). In: 20th ACM STOC. pp. 11-19. ACM Press (May 1988)

14. Chida, K., Genkin, D., Hamada, K., Ikarashi, D., Kikuchi, R., Lindell, Y., Nof, A.: Fast large-scale honest-majority MPC for malicious adversaries. In: Shacham, H., Boldyreva, A. (eds.) CRYPTO 2018, Part III. LNCS, vol. 10993, pp. 34-64. Springer, Heidelberg (Aug 2018)

15. Cramer, R., Damgård, I., Escudero, D., Scholl, P., Xing, C.: SPD $\mathbb{Z}_{2^{k}}$ : Efficient MPC mod $2^{k}$ for dishonest majority. In: Shacham, H., Boldyreva, A. (eds.) CRYPTO 2018, Part II. LNCS, vol. 10992, pp. 769-798. Springer, Heidelberg (Aug 2018)

16. Damgård, I., Fitzi, M., Kiltz, E., Nielsen, J.B., Toft, T.: Unconditionally secure constant-rounds multi-party computation for equality, comparison, bits and exponentiation. In: Halevi, S., Rabin, T. (eds.) TCC 2006. LNCS, vol. 3876, pp. 285-304. Springer, Heidelberg (Mar 2006) 
17. Damgård, I., Geisler, M., Krøigaard, M., Nielsen, J.B.: Asynchronous multiparty computation: Theory and implementation. In: Jarecki, S., Tsudik, G. (eds.) PKC 2009. LNCS, vol. 5443, pp. 160-179. Springer, Heidelberg (Mar 2009)

18. Damgård, I., Keller, M., Larraia, E., Pastro, V., Scholl, P., Smart, N.P.: Practical covertly secure MPC for dishonest majority - or: Breaking the SPDZ limits. In: Crampton, J., Jajodia, S., Mayes, K. (eds.) ESORICS 2013. LNCS, vol. 8134, pp. 1-18. Springer, Heidelberg (Sep 2013)

19. Damgård, I., Nielsen, J.B., Nielsen, M., Ranellucci, S.: The TinyTable protocol for 2-party secure computation, or: Gate-scrambling revisited. In: Katz, J., Shacham, H. (eds.) CRYPTO 2017, Part I. LNCS, vol. 10401, pp. 167-187. Springer, Heidelberg (Aug 2017)

20. Damgård, I., Pastro, V., Smart, N.P., Zakarias, S.: Multiparty computation from somewhat homomorphic encryption. In: Safavi-Naini, R., Canetti, R. (eds.) CRYPTO 2012. LNCS, vol. 7417, pp. 643-662. Springer, Heidelberg (Aug 2012)

21. Giacomelli, I., Madsen, J., Orlandi, C.: ZKBoo: Faster zero-knowledge for Boolean circuits. In: Holz, T., Savage, S. (eds.) USENIX Security 2016. pp. 1069-1083. USENIX Association (Aug 2016)

22. Ishai, Y., Kushilevitz, E., Ostrovsky, R., Sahai, A.: Zero-knowledge from secure multiparty computation. In: Johnson, D.S., Feige, U. (eds.) 39th ACM STOC. pp. 21-30. ACM Press (Jun 2007)

23. Katz, J., Kolesnikov, V., Wang, X.: Improved non-interactive zero knowledge with applications to post-quantum signatures. In: Lie, D., Mannan, M., Backes, M., Wang, X. (eds.) ACM CCS 2018. pp. 525-537. ACM Press (Oct 2018)

24. Keller, M.: MP-SPDZ: A versatile framework for multi-party computation. In: Ligatti, J., Ou, X., Katz, J., Vigna, G. (eds.) ACM CCS 20. pp. 1575-1590. ACM Press (Nov 2020)

25. Keller, M., Orsini, E., Rotaru, D., Scholl, P., Soria-Vazquez, E., Vivek, S.: Faster secure multi-party computation of AES and DES using lookup tables. In: Gollmann, D., Miyaji, A., Kikuchi, H. (eds.) ACNS 17. LNCS, vol. 10355, pp. 229-249. Springer, Heidelberg (Jul 2017)

26. Keller, M., Orsini, E., Scholl, P.: MASCOT: Faster malicious arithmetic secure computation with oblivious transfer. In: Weippl, E.R., Katzenbeisser, S., Kruegel, C., Myers, A.C., Halevi, S. (eds.) ACM CCS 2016. pp. 830-842. ACM Press (Oct 2016)

27. Rotaru, D., Wood, T.: MArBled circuits: Mixing arithmetic and Boolean circuits with active security. In: Hao, F., Ruj, S., Sen Gupta, S. (eds.) INDOCRYPT 2019. LNCS, vol. 11898, pp. 227-249. Springer, Heidelberg (Dec 2019) 